\title{
REACTION-RATE, MIXTURE-FRACTION, AND TEMPERATURE IMAGING IN TURBULENT METHANE/AIR JET FLAMES
}

\author{
JONATHAN H. FRANK, ${ }^{1}$ SEBASTIAN A. KAISER ${ }^{2}$ AND MARSHALL B. LONG ${ }^{2}$ \\ ${ }^{1}$ Combustion Research Facility \\ Sandia National Laboratories \\ Livermore, CA 94551, USA \\ ${ }^{2}$ Department of Mechanical Engineering \\ Yale University \\ New Haven, CT 06520, USA
}

\begin{abstract}
Instantaneous two-dimensional measurements of reaction rate, mixture fraction, and temperature are demonstrated in turbulent partially premixed methane/air jet flames. The forward reaction rate of the reaction $\mathrm{CO}+\mathrm{OH} \Rightarrow \mathrm{CO}_{2}+\mathrm{H}$ is measured by simultaneous $\mathrm{OH}$ laser-induced fluorescence (LIF) and two-photon CO LIF. The product of the two LIF signals is shown to be proportional to the reaction rate. Temperature and fuel concentration are measured using polarized and depolarized Rayleigh scattering. A three-scalar technique for determining mixture fraction is investigated using a combination of polarized Rayleigh scattering, fuel concentration, and CO LIF. Measurements of these three quantities are coupled with previous detailed multiscalar point measurements to obtain the most probable value of the mixture fraction at each point in the imaged plane. This technique offers improvements over two-scalar methods, which suffer from decreased sensitivity around the stoichiometric contour and biases in fuel-rich regions due to parent fuel loss. Simultaneous reaction-rate, mixture-fraction, and temperature imaging is demonstrated in laminar $(R e=1100)$ and turbulent $(R e=22,400) \mathrm{CH}_{4} /$ air $(1 / 3$ by volume $)$ jet flames. The turbulent jet flame is the subject of multiple numerical modeling efforts. A primary objective for developing these imaging diagnostics is to provide measurements of fundamental quantities that are needed to accurately model interactions between turbulent flows and flames.
\end{abstract}

\section{Introduction}

The development of multiscalar imaging diagnostics for turbulent flames is essential to understanding the interaction of turbulent flows and flames. A central objective in diagnostic research is to develop techniques for instantaneous multidimensional measurements of fundamental quantities, such as reaction rate and mixture fraction. These quantities of interest can be determined at a single point using multiscalar measurements, which are widely available [1-3] and can provide as many as 10 simultaneous measurements, including temperature and nine species [1]. However, only a subset of these scalars can be measured simultaneously in two dimensions. The challenge in imaging diagnostics is to measure fundamental quantities of interest by combining a judicious choice of laser-based imaging techniques. In the present work, we focus on combined laser-induced fluorescence (LIF) and Rayleigh measurements to determine the reaction rate and mixture fraction in two dimensions.

\section{Reaction-Rate Imaging}

Recently, the feasibility of reaction-rate imaging has been demonstrated in laminar premixed flames by combining two LIF measurements [4-6]. The basic concept is described here using the reaction $\mathrm{CO}+$ $\mathrm{OH} \Rightarrow \mathrm{CO}_{2}+\mathrm{H}$, which is the dominant reaction pathway for $\mathrm{CO}_{2}$ production in $\mathrm{CH}_{4} /$ air flames and is a focus of the present work. The forward reaction rate, $\mathrm{RR}$, is given by $\mathrm{RR}=k(T)[\mathrm{CO}][\mathrm{OH}]$, where $k(T)$ is the forward rate constant and $T$ is the temperature. The basis of this diagnostic involves using the product of simultaneous OH LIF and two-photon CO LIF measurements to obtain a signal that is proportional to the reaction rate. The product of LIF signals from $\mathrm{CO}$ and $\mathrm{OH}$ can be approximated by $f_{\mathrm{CO}}(T) f_{\mathrm{OH}}(T)[\mathrm{CO}][\mathrm{OH}]$, where the temperature dependence of the LIF signals is represented by $f(T)$. The exact functional dependence of $f(T)$ depends on the particular transition(s) employed, the spectral characteristics of the detection system, and the temperature dependence of the quenching cross section. For reaction-rate imaging, the strategy is to select pump/detection schemes such that $f_{\mathrm{OH}}(T) f_{\mathrm{CO}}(T) \propto k(T)$. When this relationship is achieved, the product of $\mathrm{CO}$ and OH LIF signals is proportional to $k(T)[\mathrm{CO}][\mathrm{OH}]$.

Previously, reaction-rate imaging was applied to $\mathrm{OH} / \mathrm{CH}_{2} \mathrm{O}[6,7]$ and $\mathrm{CO} / \mathrm{OH}[4,5]$ combinations. 


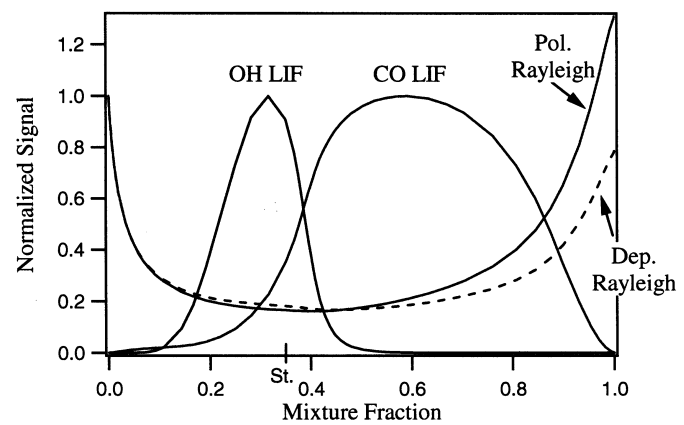

FIG. 1. Predicted profiles of signal versus mixture fraction for polarized and depolarized Rayleigh scattering, $\mathrm{OH}$ $\mathrm{LIF}$, and two-photon CO LIF in a partially premixed $\mathrm{CH}_{4} /$ air flame with a strain rate of $100 \mathrm{~s}^{-1}$.

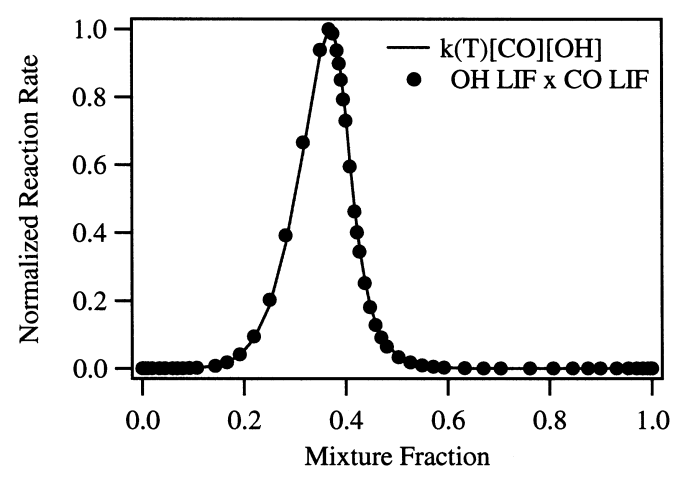

FIG. 2. Comparison of normalized reaction rate and the product of predicted $\mathrm{OH}$ LIF and CO LIF signals as a function of mixture fraction.

The previous demonstrations of $\mathrm{CO}+\mathrm{OH}$ reactionrate imaging were limited to repeatable flow-flame interactions in laminar premixed flames. In those studies, phase averaging was used to improve the signal-to-noise ratios of two-photon CO LIF, which is a relatively weak process compared to single-photon LIF. Attempts to increase the CO LIF signal for imaging include the use of a multipass cell [8] and broadband collection [5]. Multipass cells are subject to beam-steering effects and are not conducive to combining multiple imaging diagnostics. Broadband collection proved to be problematic because of lasergenerated interference from $\mathrm{C}_{2}{ }^{*}$ emission [5]. Subtraction of this interference required a separate offresonance measurement. This approach is not practical for instantaneous measurements in turbulent flames. In the broadband detection scheme, the $\mathrm{C}_{2}{ }^{*}$ emission can be a significant fraction of the total CO LIF signal in rich premixed flames and can overwhelm the CO LIF signal in nonpremixed or partially premixed flames.
In the present work, we investigate reaction-rate imaging of $\mathrm{CO}+\mathrm{OH}$ in partially premixed $\mathrm{CH}_{4} /$ air jet flames using a narrow bandpass filter to eliminate $\mathrm{C}_{2}{ }^{*}$ emission. Fig. 1 shows predicted profiles of $\mathrm{CO}$ $\mathrm{LIF}$ and OH LIF as a function of mixture fraction for a partially premixed laminar $\mathrm{CH}_{4} /$ air $(1 / 3$ by volume) flame using a laminar flame calculation with a strain rate of $100 \mathrm{~s}^{-1}$ [9]. In partially premixed and nonpremixed methane flames, the $\mathrm{OH}$ peaks on the lean side of the reaction zone, and the CO peaks on the rich side. In Fig. 1, the two profiles overlap for a narrow range of mixture fraction bracketing the stoichiometric value of $f_{\text {st }}=0.351$.

First, we examine the feasibility of choosing a pump/detection scheme for $\mathrm{OH}$ and CO LIF such that $f_{\mathrm{OH}}(T) f_{\mathrm{CO}}(T) \propto k(T)$. Ideally, the two-photon CO LIF should be excited near the peak of the excitation spectrum in the flame of interest since it is a relatively weak scattering process. Calculations for the partially premixed flames considered here showed that optimal pumping is achieved for overlapping Q-branch transitions in the B-X $(0,0)$ Hopefield-Birge system. The $\mathrm{OH}$ pumping scheme was subsequently chosen to best obtain the proportionality, $f_{\mathrm{OH}}(T) f_{\mathrm{CO}}(T) \propto k(T)$. This was achieved by excitation of the $\mathrm{Q}_{1}(12)$ transition of the $\mathrm{A}-\mathrm{X}(1,0)$ band. The temperature dependence of the product $f_{\mathrm{OH}}(T) f_{\mathrm{CO}}(T)$ for these pump schemes was compared with $k(T)$, using $k(T)=B T^{\mathrm{M}} \mathrm{e}^{-E / R T}$, where $T$ is temperature, $B=4.76 \times 10^{7} \mathrm{~mol} / \mathrm{cm}^{3}, M=1.23$, and $E=-293 \mathrm{~J} / \mathrm{mol}$ (GRI-Mech 2.11). Over the temperature range of significant $\mathrm{CO}+\mathrm{OH}$ reaction $(1600-1950 \mathrm{~K})$, the rate constant, $k(T)$, is not a strong function of temperature and only varies by slightly more than $\pm 10 \%$.

The proportionality of $f_{\mathrm{OH}}(T) f_{\mathrm{CO}}(T)$ to $k(T)$ is excellent between 1600 and $1800 \mathrm{~K}$, but the value of $f_{\mathrm{OH}}(T) f_{\mathrm{CO}}(T)$ deviates from $k(T)$ by as much as $12 \%$ at temperatures above $1800 \mathrm{~K}$ and becomes a dualvalued function of temperature. In nonpremixed or partially premixed flames, $f_{\mathrm{OH}}(T) f_{\mathrm{CO}}(T)$ is dual valued because of different quenching environments for lean and rich mixture fractions. In general, this presents a challenge when trying to attain the necessary proportionality with $k(T)$. Fortunately, this is not a significant limitation for the $\mathrm{CO}+\mathrm{OH}$ reaction-rate diagnostic since $k(T)$ is not a strong function of temperature, and pumping schemes can be chosen such that $f_{\mathrm{OH}}(T) f_{\mathrm{CO}}(T)$ also has a relatively weak temperature dependence over the relevant range of temperature.

Figure 2 shows a comparison of predicted profiles of reaction rate and the product of $\mathrm{CO} \mathrm{LIF}$ and $\mathrm{OH}$ LIF. Both profiles have been normalized to their peak values. The figure demonstrates that the product of LIF signals quite accurately represents the reaction rate. The bounds on the predicted systematic error in using the product of LIF signals to measure reaction rate are approximately $8.1 \%$ on the 
lean side and $-5.5 \%$ on the rich side. The lean-side errors are greater because of the larger temperature range over which the $\mathrm{CO}+\mathrm{OH}$ reaction is significant. For this same reason, the lean side is more sensitive to systematic errors introduced by less optimal choices of $\mathrm{CO}$ and $\mathrm{OH}$ excitation schemes.

\section{Mixture-Fraction Imaging}

The mixture fraction can be determined by measuring all major species. However, it is impractical to do this in two dimensions. Mixture-fraction imaging thus requires the identification of a subset of these measurements that can provide an accurate measure of the mixture fraction with enough signal for two-dimensional measurements. Previous efforts have focused on a two-scalar approach that combines Rayleigh scattering and fuel concentration measurements. A fundamental difficulty with this approach is that it is not very sensitive near stoichiometric conditions, where the fuel signal disappears and the Rayleigh signal does not vary greatly as a function of mixture fraction. Methods for obtaining fuel concentration have included LIF of fuel tracers $[10,11]$, Raman scattering from fuel $[10-13]$, and difference Rayleigh scattering [14]. The most promising of these techniques is difference Rayleigh scattering, which is used in the present experiments.

In difference Rayleigh scattering, temperature and fuel measurements are taken by simultaneously recording polarized and depolarized components of Rayleigh scattering. The details of this technique are described elsewhere [14], and only a brief overview is provided here. The depolarized Rayleigh scattering is a function of the effective depolarization ratio for the local composition. Isotropic molecules, such as methane, have an extremely small depolarization ratio. In a methane/air flame, regions containing methane have a reduced depolarization ratio. When normalized to the signal in air, the difference between the polarized and depolarized signal provides a measure of fuel concentration.

In the present work, we investigate the use of a three-scalar technique to obtain the mixture fraction. The third scalar, CO, is a relatively strong function of mixture fraction near stoichiometric conditions and provides improved sensitivity for determining the mixture fraction (Fig. 1). Previously, one other three-scalar technique has been investigated using $\mathrm{N}_{2}$ Raman imaging [15]. This method provided relatively modest signal-to-noise ratios and required the substitution of argon for $\mathrm{N}_{2}$ in partially premixed fuel/air mixtures to obtain sensitivity near the stoichiometric contour.

\section{Experimental Methods}

Experiments were performed in the Advanced Imaging Laboratory at Sandia's Combustion Research Facility using the experimental apparatus shown in Fig. 3. A combination of four lasers and five cameras was used to simultaneously image $\mathrm{OH}$ LIF, two-photon CO LIF, and polarized and depolarized Rayleigh scattering.

\section{$\mathrm{OH}$ and $\mathrm{CO}$ Measurements}

Two-dimensional measurements of $\mathrm{OH}$ and twophoton CO LIF were performed simultaneously. For OH LIF, the frequency-doubled output from a Nd:YAG-pumped dye laser was tuned near $285 \mathrm{~nm}$ to pump the $\mathrm{Q}_{1}(12)$ transition of the A-X $(1,0)$ band. The $\mathrm{OH}$ fluorescence from the $(0,0)$ and $(1,1)$ bands was reflected by a dichroic beam splitter with a reflective coating from 300 to $350 \mathrm{~nm}$ and imaged onto an intensified CCD camera (Andor Technology, $512 \times 512$ pixels) with an $f / 1.8$ Cerco quartz camera lens. The projected pixel size was $67 \mu \mathrm{m} \times 67 \mu \mathrm{m}$. The image intensifier was gated for $400 \mathrm{~ns}$, bracketing the dye laser pulse and eliminating any interference from the other lasers. The OH LIF images were corrected for spatial variations in the laser sheet using acetone LIF to record the beam profile.

Two-photon excitation of overlapping transitions in the $\mathrm{B}-\mathrm{X}(0,0)$ Hopefield-Birge system of $\mathrm{CO}$ was achieved using the frequency-doubled output from a Nd:YAG-pumped optical parametric oscillator (OPO) (22 mJ) near $230.1 \mathrm{~nm}$. The laser was tuned to maximize the CO LIF signal in a laminar partially premixed $\mathrm{CH}_{4} /$ air (1/3 by volume) flame. Sheetforming optics were used to form a 6-mm high laser sheet. The two-photon excitation scheme for $\mathrm{CO}$ required a high-intensity laser sheet to maximize the CO LIF signal. The average laser beam profile was measured using CO LIF from a dilute mixture of $\mathrm{CO}$ in $\mathrm{N}_{2}(0.3 \%$ CO by volume). Shot-to-shot fluctuations in the laser sheet profile were recorded on an unintensified CCD camera. The OPO beam profile was sampled by a fused silica wedge positioned after the sheet-forming optics. Filters placed in front of the beam-profile camera transmitted the 230-nm beam while blocking light from the other lasers. Corrections for shot-to-shot fluctuations were important for the CO LIF measurements because of the sensitivity of two-photon CO LIF to variations in beam intensity.

Timing of the laser pulses was controlled with digital delay generators. The lasers fired sequentially, with the dye laser firing first and the OPO following after a 470-ns delay. The sequential timing of the lasers and intensifiers eliminated the possibility of cross talk between the two diagnostics. The joint $\mathrm{CO} / \mathrm{OH}$ LIF measurements were essentially instantaneous because the elapsed time for a single measurement was orders of magnitude less than the flow timescales.

The CO fluorescence was reflected by a dichroic beam splitter and imaged onto an intensified CCD camera (Andor Technology, $512 \times 512$ pixels). The 


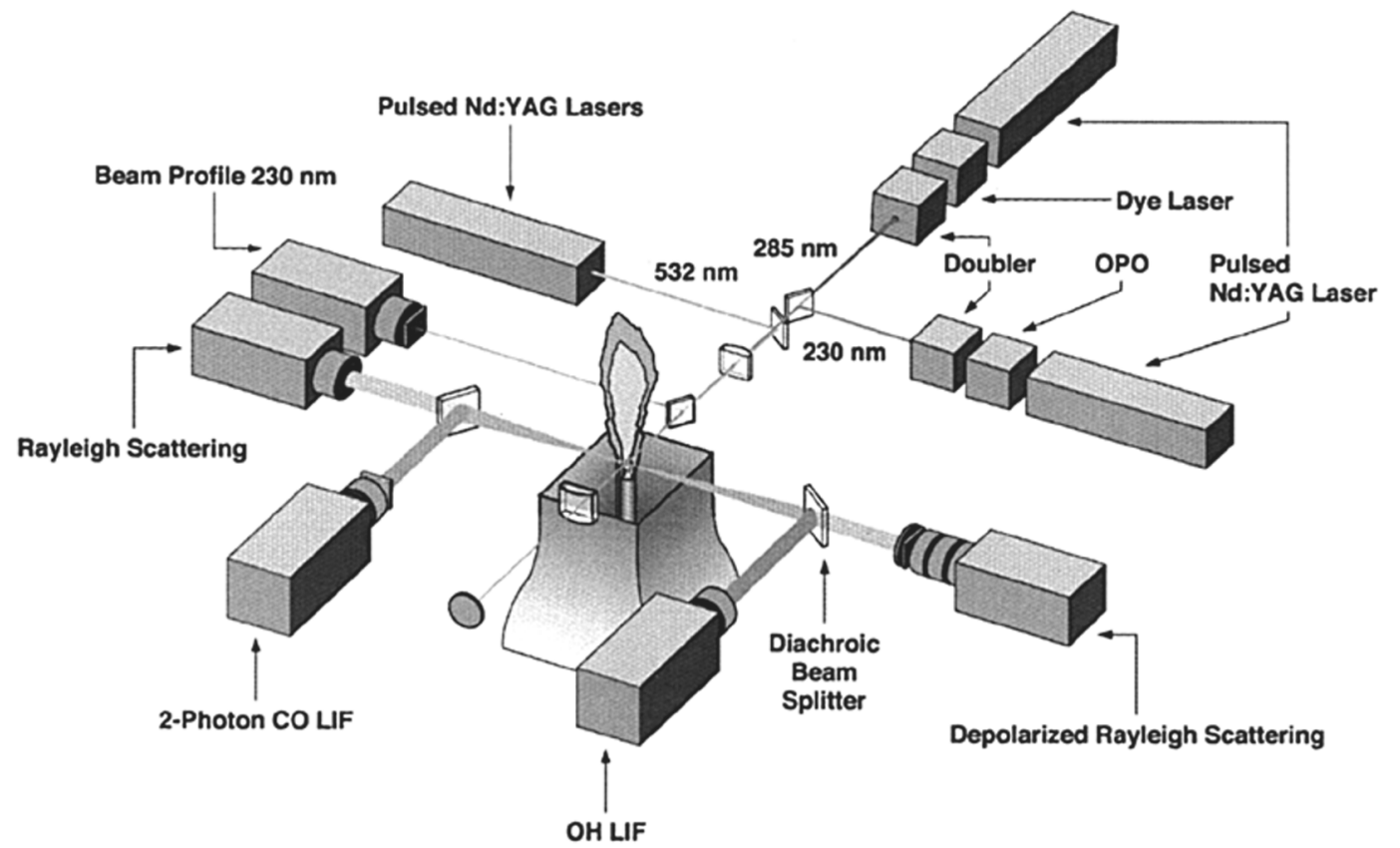

FIG. 3. Experimental setup for simultaneous imaging of polarized and depolarized Rayleigh scattering, OH LIF, and two-photon CO LIF.

imaging system included an $f / 1.2$ camera lens and a narrow bandpass interference filter $\left(\lambda_{\text {center }}=484 \mathrm{~nm}\right.$, $\Delta \lambda=10 \mathrm{~nm})$, which transmitted fluorescence from the B-A $(0,1)$ transition at $483.5 \mathrm{~nm}$ and blocked laser-generated Swan band emission from $\mathrm{C}_{2}{ }^{*}$. The projected pixel size was $71 \mu \mathrm{m} \times 71 \mu \mathrm{m}$. The image intensifier for the CO LIF camera was gated for $400 \mathrm{~ns}$, bracketing the OPO laser pulse. The singleshot signal-to-noise ratio in the jet flames was approximately 15 for the unsmoothed CO LIF signal. A Gaussian smoothing kernel $\left(\sigma_{x}=\sigma_{y}=90 \mu \mathrm{m}\right)$ was used to further improve the signal-to-noise ratio in the data analysis.

\section{Temperature and Fuel Measurements}

Temperature and fuel measurements were performed using joint polarized/depolarized Rayleigh scattering. The depolarized component of Rayleigh scattering is typically 2 orders of magnitude smaller than polarized Rayleigh scattering. To provide sufficient signal for depolarized Rayleigh imaging, two vertically polarized Nd:YAG lasers (360 and $560 \mathrm{~mJ}$ / pulse at $532 \mathrm{~nm}$ ) were spatially overlapped and separated temporally by $110 \mathrm{~ns}$. The first of the Nd:YAG lasers was fired $416 \mathrm{~ns}$ prior to the dye laser used for OH LIF. The two Nd:YAG beams were formed into coaligned sheets within the imaged region. A retroreflection of both beams was used to further increase signal. After the first pass through the test section, the beams were collimated with a cylindrical lens and retroreflected by a flat mirror. On the return path, the beams were focused to a sheet by a second pass through the cylindrical lens.

Vertically polarized Rayleigh scattering was imaged onto an unintensified interline transfer CCD camera (Sensicam, $640 \times 512$ pixels after $2 \times 2$ binning) with a projected pixel dimension of $53 \mu \mathrm{m}$ $\times 53 \mu \mathrm{m}$ and an exposure period of $600 \mathrm{~ns}$, bracketing both Nd:YAG lasers. Rayleigh scattering was transmitted through a dichroic beamsplitter and collected with an f/1.4 50-mm focal length camera lens. On the opposite side of the burner, the horizontal component of Rayleigh scattering was imaged onto an intensified CCD camera (Sensicam, $320 \times 240$ pixels after $2 \times 2$ binning) with a projected pixel dimension of $90 \mu \mathrm{m} \times 90 \mu \mathrm{m}$. Rayleigh scattering was transmitted through a dichroic beamsplitter and collected with an $f / 1.285-\mathrm{mm}$ focal length camera lens. A polarizer $(\mathrm{B}+\mathrm{W}$ photographic circular polarizer) transmitted the horizontally polarized Rayleigh scattering and blocked the vertically polarized component. A narrow bandpass filter ( $\lambda_{\text {center }}=532 \mathrm{~nm}, \Delta \lambda=10 \mathrm{~nm}$ ) eliminated broadband laser-generated interference. The intensifier was gated for $400 \mathrm{~ns}$, bracketing both Nd:YAG laser pulses. The single-shot signal-to-noise ratios in ambient air were approximately 10 and 75 for the unsmoothed depolarized and polarized Rayleigh signals, respectively. Contour-aligned smoothing was 


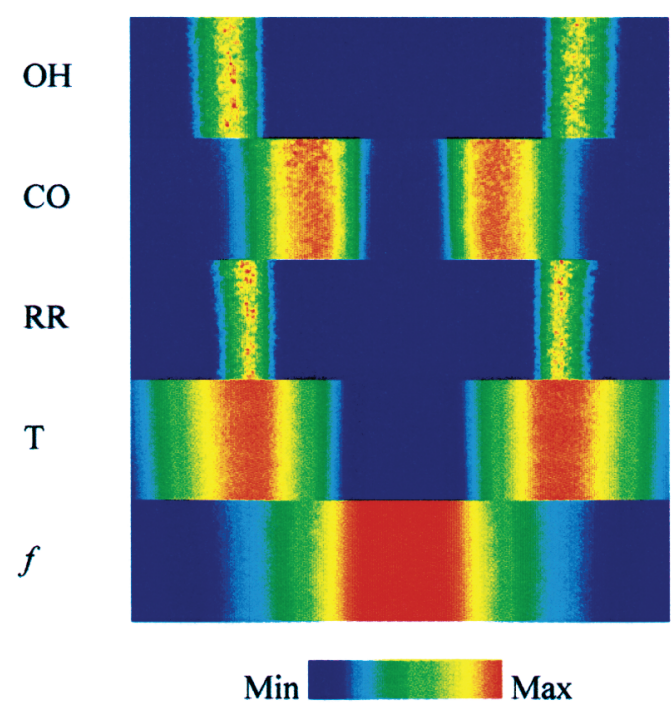

FIG. 4. Simultaneous measurements of CO LIF, OH $\mathrm{LIF}$, reaction rate $(\mathrm{RR})$, temperature $(T)$, and mixture fraction $(f)$ in a laminar $\mathrm{CH}_{4}$ /air jet flame. Images are centered at $x / d=5$ and are 40 -shot averages.

used to further improve the signal-to-noise ratio of the depolarized Rayleigh signal.

\section{Image Matching and Stripe Corrections}

A precise image-matching technique was developed to obtain accurate registration between the CCD cameras. The polarized Rayleigh camera, which had the highest spatial resolution, was used as the reference to which the other three images were matched. The relative displacement between cameras was determined at 200 locations throughout the imaged region using a cross-correlation analysis of target images. Images were matched with an eightparameter bilinear geometric warping algorithm. The eight parameters were determined from a regression of the 200 displacement vectors. The residual matching error was in the subpixel range.

When laser beams propagate through flames, beam steering can be noticeable over extended path lengths. The dual-pass arrangement used for Rayleigh scattering was sensitive to beam-steering effects, which arose from gradients in the index of refraction associated with density variations in the flame. Beam steering introduced stripes in the laserbeam profile, and these stripes fluctuated from shot to shot as the temperature profile varied in the turbulent flame. To correct for these stripes, the Nd:YAG beam profile was recorded for each shot by including a region of ambient air in the polarized Rayleigh image. However, a correction with a singlebeam profile did not sufficiently remove stripes across the entire imaged region because the stripes were inherently not parallel. Residual stripes were identified using an image analysis algorithm, and a second stage of correction significantly reduced the impact of beam steering.

\section{Results and Discussion}

Simultaneous two-dimensional measurements of reaction rate, temperature, and fuel are demonstrated in laminar and turbulent axisymmetric jet flames. These flames correspond to flames A and D in the TNF Workshop library and are the subject of multiple numerical modeling efforts [16]. Detailed descriptions, including single-point measurements of species, temperature, and velocity, are available via the internet [16] and in Refs. $[1,17,18]$.

\section{Laminar Flame}

A partially premixed axisymmetric laminar flame with the same fuel composition as the turbulent flame was used as a test case for verifying the mixture-fraction and reaction-rate measurement techniques. A steady laminar $\mathrm{CH}_{4}$ /air (1/3 by volume) jet flame with $R e=1100$ was stabilized on a $7.2-\mathrm{mm}$ diameter nozzle. Fig. 4 shows results of simultaneous two-dimensional measurements over a $21-\mathrm{mm}$ wide region centered on the jet axis at $x / d=5$. The images are an average of 40 single shots and have been cropped to show only the region that is common to all four imaging diagnostics. The $\mathrm{CO}$ and $\mathrm{OH}$ LIF signals are displayed without any corrections for collisional quenching or Boltzmann fraction variations because the main focus here is to use the LIF signals for determining the reaction rate. The relatively broad CO profile peaks in the fuel-rich region and is surrounded by a layer of $\mathrm{OH}$, which peaks to the lean side of stoichiometric and slightly overlaps the region of CO. The reaction-rate image was determined from the pixel-by-pixel product of the $\mathrm{CO}$ and OH LIF images, and the reaction-rate peak is observed to coincide with the location of maximum temperature. The mixture-fraction and temperature images indicate a 3.3-mm wide potential core of undiluted fuel/air mixture near the jet axis.

The mixture fraction was determined using a combination of depolarized and polarized Rayleigh scattering and CO LIF. The functional dependence of each signal on mixture fraction was determined from detailed single-point measurements [1]. The polarized Rayleigh signal has the highest signal-to-noise ratio and provides the best measure of the mixture fraction at both extremes of mixture fraction (below $\sim 0.2$ and above $\sim 0.5$ ). However, the Rayleigh signal is a relatively weak function of mixture fraction near stoichiometric, where $\mathrm{CO}$ can provide improved sensitivity. In addition, the Rayleigh signal by itself 


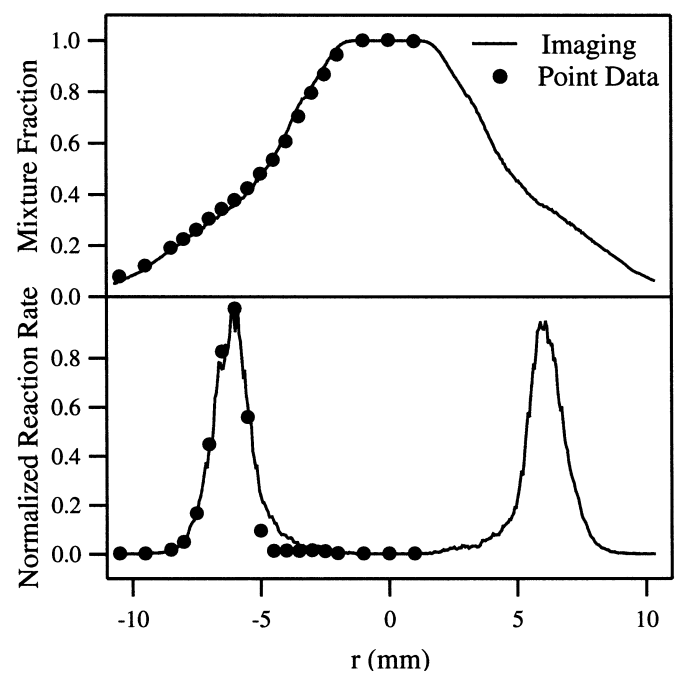

FIG. 5. Comparison of radial profiles from imaging (line) and multiscalar single-point measurements (circles) of mixture fraction (top) and reaction rate (bottom) in a laminar $\mathrm{CH}_{4} /$ air jet flame at $x / d=5$.

cannot be used to determine the mixture fraction because it is a dual-valued function of mixture fraction, as seen in Fig. 1. This ambiguity can be eliminated by using measurements of CO LIF and difference Rayleigh imaging to identify the lean and rich regions of the jet flame. Difference Rayleigh measurements were used to identify fuel-rich regions with a mixture fraction greater than 0.5 . For mixture fraction values between 0.5 and 0.2 , the CO LIF was used to indicate whether the local mixture was fuel rich or lean. Regions with a mixture fraction less than 0.2 were identified by the lack of both fuel and $\mathrm{CO}$.

Detailed single-point measurements of species and temperature in this laminar flame provide a means to validate the mixture-fraction and reactionrate imaging techniques [1]. The top panel of Fig. 5 shows a comparison of radial profiles of mixture fraction. The solid line shows the radial profile of the mixture fraction from Fig. 4. The profile has been averaged in the axial direction over a 5-mm region centered at $x / d=5$. The circles indicate values of the mixture fraction determined from multiscalar point measurements using the following more complete formulation of mixture fraction $[1,19]$ :

$$
f=\frac{2\left(Y_{\mathrm{C}}-Y_{\mathrm{C}, 2}\right) / w_{\mathrm{C}}+\left(Y_{\mathrm{H}}-Y_{\mathrm{H}, 2}\right) / 2 w_{\mathrm{H}}}{2\left(Y_{\mathrm{C}, 1}-Y_{\mathrm{C}, 2}\right) / w_{\mathrm{C}}+\left(Y_{\mathrm{H}, 1}-Y_{\mathrm{H}, 2}\right) / 2 w_{\mathrm{H}}}
$$

where Y's are elemental mass fractions, $w$ 's are atomic weights, and subscripts 1 and 2 refer to the main jet and coflowing air stream, respectively. The mixture-fraction imaging technique shows excellent agreement with the point measurements and demonstrates the successful implementation of the scheme for determining the mixture fraction.

A comparison of the radial profiles of reaction-rate is shown in the bottom panel of Fig. 5. The solid line is a radial profile of the normalized product of $\mathrm{CO}$ and $\mathrm{OH}$ LIF shown in the reaction-rate image of Fig. 4 . The circles show the reaction rate determined from $\mathrm{RR}=k(T)[\mathrm{OH}][\mathrm{CO}]$, where the temperature and $\mathrm{OH}$ and $\mathrm{CO}$ concentrations from the multiscalar point measurements are used and the rate constant parameters are taken from GRI-Mech 2.11. Overall, the reaction-rate profiles match quite well, indicating that reaction-rate imaging is quite accurate in these partially premixed jet flames. The reaction-rate imaging shows too high a reaction rate on the fuelrich side for radial locations between approximately 4.0 and $5.0 \mathrm{~mm}$. This location corresponds to mixture fraction values between 0.5 and 0.6 , where polycyclic aromatic hydrocarbon interference has been observed in single-point Raman measurements [20]. The addition of a narrow bandpass filter could reduce the interference.

\section{Turbulent Flame Results}

The turbulent flame considered here is a turbulent $\mathrm{CH}_{4} /$ air ( $1 / 3$ by volume) piloted jet flame with $R e=22,400$. The $7.2-\mathrm{mm}$ diameter main jet was surrounded by an 18.2-mm diameter pilot, which helped anchor the turbulent flame. The pilot flame was comprised of a mixture of $\mathrm{C}_{2} \mathrm{H}_{2}, \mathrm{H}_{2}$, air, $\mathrm{CO}_{2}$, and $\mathrm{N}_{2}$ with the same enthalpy and equilibrium composition as a $\mathrm{CH}_{4} /$ air flame with an equivalence ratio of 0.77 . Detailed single-point measurements were used to determine the functional dependence of each measured signal on the mixture fraction [1]. The point measurements indicate that at stoichiometric conditions, the root mean square fluctuations of the Rayleigh signal are approximately $7 \%$.

The images in Fig. 6 show two separate instantaneous measurements of $\mathrm{CO}$ and $\mathrm{OH} \mathrm{LIF}$, reaction rate, temperature, and mixture fraction at a downstream location of $x / d=15$. The jet centerline is located on the right side of each image, where the smallest scale turbulent structures are seen in the unreacted fuel/air mixture. The reaction zone is a thin strip located near the stoichiometric mixturefraction contour.

The temperature and mixture-fraction measurements provide a means to verify the reaction-rate diagnostic technique, since the reaction rate can be determined by $k(T)[\mathrm{OH}][\mathrm{CO}]$ and compared with the product of the LIF signals. For this purpose, the $\mathrm{OH}$ and CO LIF images were converted to concentrations by using species and temperature data from single-point measurements to determine quenching rates as a function of mixture fraction [1]. Quenching cross sections for $\mathrm{OH}$ were obtained from Ref. [21]. 


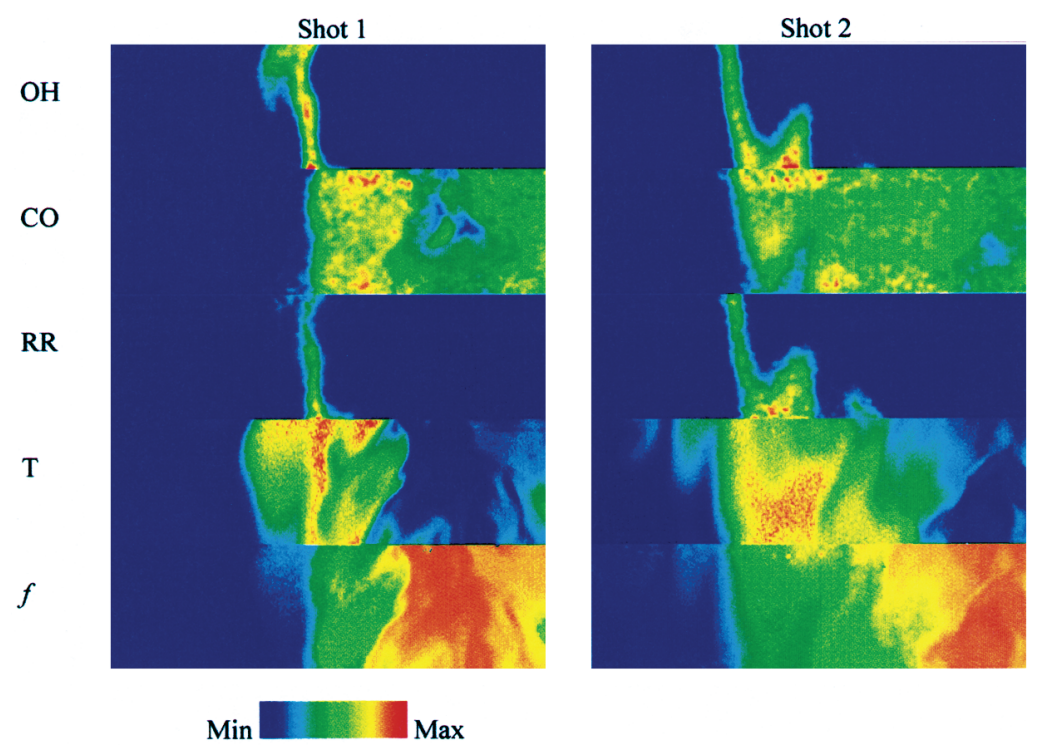

FIG. 6. Simultaneous single-shot measurements of OH LIF, CO LIF, reaction rate (RR), temperature $(T)$, and mixture fraction $(f)$ in a turbulent $\mathrm{CH}_{4}$ /air jet flame. Images are centered at $x / d=15$.

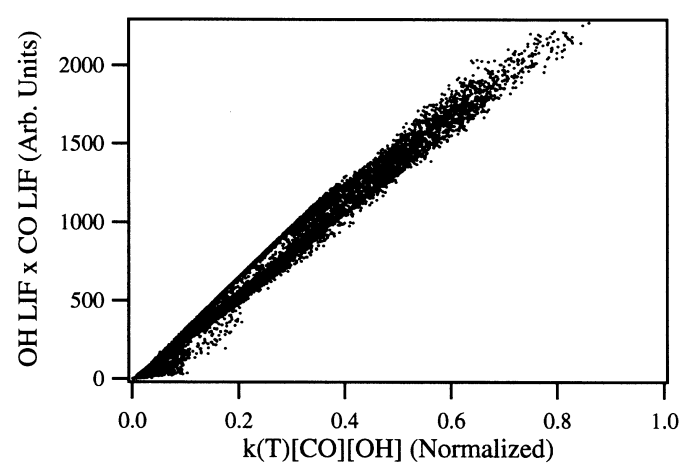

FIG. 7. Correlation of $\mathrm{OH} \mathrm{LIF} \times \mathrm{CO} \mathrm{LIF}$ with reaction rate determined from $k(T)[\mathrm{OH}][\mathrm{CO}]$ in turbulent partially premixed $\mathrm{CH}_{4} /$ air jet flame.

Recent measurements of temperature-dependent quenching cross sections of two-photon CO LIF [22] were used to determine $\mathrm{CO}$ concentrations.

As a check on the diagnostic technique, the reaction rate was determined both by multiplying $\mathrm{CO}$ and $\mathrm{OH}$ LIF signals and by $k(T)[\mathrm{OH}][\mathrm{CO}]$ for shot 2 in Fig. 6. Fig. 7 shows the excellent correlation between the two methods, indicating that the product of LIF signals provides a good measure of the reaction rate in these flames.

\section{Conclusions}

Simultaneous two-dimensional measurements of reaction rate, temperature, and fuel were demonstrated in laminar and turbulent partially premixed
$\mathrm{CH}_{4}$ /air jet flames using a combination of two-photon CO LIF, OH LIF, and depolarized and polarized Rayleigh scattering. The instantaneous forward reaction rate for $\mathrm{CO}+\mathrm{OH} \Rightarrow \mathrm{CO}_{2}+\mathrm{H}$ was determined from the pixel-by-pixel product of $\mathrm{CO}$ and $\mathrm{OH}$ LIF images.

The CO LIF combined with depolarized and polarized Rayleigh scattering provided a three-scalar technique for unambiguously determining the mixture fraction. Multiscalar single-point measurements were used to determine the functional dependence of polarized Rayleigh scattering and CO LIF on mixture fraction. These functions were then used to determine the instantaneous mixture fraction. The difference Rayleigh and CO LIF were used to identify fuel-rich and fuel-lean regions. This eliminated the ambiguity of using the dual-valued Rayleigh signal alone to determine the mixture fraction. The threescalar mixture-fraction imaging technique is an improvement to two-scalar methods, which suffer from decreased sensitivity near the stoichiometric contour and biases in fuel-rich regions due to parent fuel loss.

\section{Acknowledgments}

This research was supported by the U.S. Department of Energy, Office of Basic Energy Sciences, Division of Chemical Sciences. The authors thank R. Sigurdsson for valuable assistance in the laboratory.

\section{REFERENCES}

1. Barlow, R. S., and Frank, J. H., Proc. Combust. Inst. 27:1087-1095 (1998). 
2. Meier, W., Vyrodov, A. O., Bergmann, V., and Stricker, W., Appl. Phys. B. 63:79-90 (1996).

3. Hassel, E. P., Appl. Opt. 32:4058-4065 (1993).

4. Frank, J. H., and Najm, H. N., "Temporal Evolution of Reaction Rate in a Vortex-Flame Interaction," paper 248, U.S. Sections Joint Meeting of the Combustion Institute, Oakland, CA, March 25-28 2001.

5. Rehm, J. E., and Paul, P. H., Proc. Combust. Inst. 28:1775-1782 (2000).

6. Paul, P. H., and Najm, H. N., Proc. Combust. Inst. 27:43-50 (1998).

7. Böckle, S., Kazenwadel, J., Kunzelmann, T., Shin, D.-I., Schulz, C., and Wolfrum, J., Proc. Combust. Inst. 28:279-286 (2000).

8. Seitzman, J. M., Haumann, J., and Hanson, R. K., Appl. Opt. 26:2892-2899 (1987).

9. Chen, J.-Y., University of California-Berkeley, personal communication, 2000.

10. Frank, J. H., Lyons, K. M., Marran, D. F., Long, M. B., Stårner, S. H., and Bilger, R. W., Proc. Combust. Inst. 25:1159-1166 (1994).

11. Long, M. B., Frank, J. H., Lyons, K. M., Marran, D. F., and Stårner, S. H., Int. J. Phys. Chem. 97:1555 (1993).

12. Stårner, S. H., Bilger, R. W., Lyons, K. M., Frank, J. H., and Long, M. B., Combust. Flame 99:347 (1994).
13. Kelman, J. B., and Masri, A. R., Combust. Sci. Technol. 129:17-55 (1997).

14. Fielding, J., Frank, J. H., Kaiser, S. A., and Long, M. B., Proc. Combust. Inst. 29:2703 (2002).

15. Fielding, J., Schaffer, A. M., and Long, M. B., Proc. Combust. Inst. 27:1007-1014 (1998).

16. International Workshop on Measurement and Computation of Turbulent Nonpremixed Flames, Sandia National Laboratories, Livermore, CA, Updated 2002, www.ca.sandia.gov/tdf/Workshop.html.

17. Masri, A. R., Dibble, R. W., and Barlow, R. S., Prog. Energy Combust. Sci. 22:307-362 (1996).

18. Stårner, S. H., Bilger, R. W., and Barlow, R. S., Combust. Sci. Technol. 70:111-133 (1990); 72:255-269 (1990).

19. Bilger, R. W., Stårner, S. H., and Kee, R. J., Combust. Flame 80:135-149 (1990).

20. Barlow, R. S., Carter, C. D., and Pitz, R. W., in Applied Combustion Diagnostics (K. Kohse-Hoeinghaus and J. Jeffries, eds.), Taylor and Francis, New York, 2002, pp. 384-407.

21. Garland, N. L., and Crosley, D. R., Proc. Combust. Inst. 21:1693-1702 (1986).

22. Settersten, T. B., Dreizler, A., and Farrow, R. L., J. Chem. Phys. 117:3173 (2002). 